\title{
THERMAL BURDEN OPTIMIZATION ON CB PROTECTIVE SUITS USING NEW MATERIALS AND INNOVATIVE DESIGN
}

\author{
Claudiu LĂZĂROAIE, Florentina ALEXE, Ciprian SĂU \\ Scientific Research Center for CBRN Defense and Ecology, Bucharest, Romania \\ claza0@yahoo.com
}

\begin{abstract}
A new mission needs new or adapted equipment. Being necessary to operate more and more frequently in warm climates and considering the new requirements for chemical and biological (CB) protective suits, it was necessary to study new materials and concepts of operation for the existing $C B$ air permeable suits. The paper presents the new requirements for reduced thermal burden protective suits, the tools for the evaluation of thermal burden and also some results of the conducted researches. The experimental results led us to a new concept in designing a suit for CB protection and also to produce a prototype batch.
\end{abstract}

\section{Keywords: thermal burden, air permeable protective suit, $\mathrm{CB}$ protection}

\section{Introduction}

The military equipment of the modern armies includes more and more efficient individual protection elements. The rapid modernization of the Romanian army, according with the NATO standard, has an important component in increasing the individual protection of the personnel who operates in CBRN contaminated environments.

Because of the variety of threats faced not only by the specialized units involved in chemical, biological, radiological and nuclear (CBRN) defense, they need good equipment on protection.

In areas with warm climate, where nowadays many operations are performed, the efficiency of CBRN-protective clothing is important, but also the comfort of the suit (expressed by reduced thermal burden to the user) is very important. A high thermal burden will significantly reduce operational efficiency and the operational tempo.

In terms of the CBRN protection equipment, there must be a choice between the isolating suits, made from various elastomers or composite materials, which provide protection against $\mathrm{CBRN}$ agents in every state (aerosols, droplets or vapors) and air permeable suits, which provide good protection against aerosols and vapors, but low protection against droplets of CBRN agents.

In terms of thermal comfort, the heat exchange between the human body and the environment is realized through skin; this exchange depends on the temperature, humidity and radiation of the skin and, on the other hand, on the air temperature, humidity and wind speed, but also on the external medium radiation received by the organism.

The heat exchange process can be approached "physically", using physiological parameters (skin temperature, vasodilatation, transpiration) which modify the heat exchanger (skin) characteristics, depending on the necessity of maintaining the organism's thermal equilibrium.

The advantage of air permeable suits consists in a lot lower thermal stress, compared with the isolating suits. In the 
operational conditions of the last years, in which the focus was on the mission completion and not on any cost protection, air permeable suits becomes the standard CBRN protection equipment for all modern armies, only poor countries (who cannot afford to spend money on endowment programs with such equipments) are still using the old fashion isolating suits.

\section{New requirements}

In 2014, Romania accepted and implemented standard STANAG 4563 "Tropical Field Clothing System" which requires a number of features that must be provided to military equipment, in order to be used in tropical climates.

According to this standard [2], the textile materials used in warm climates must have, among others, the following characteristics:

- light weight, high air permeability and satisfactory tear strength;

- low water vapor resistance.

Furthermore, in terms of design, a maximum wear comfort shall be achieved by considerable ventilation openings, in areas with heat accumulations, for optimum air circulation and freedom of movement.

Therefore, in order to optimize air permeable $\mathrm{CB}$ protective suits in terms of thermal burden two factors must be

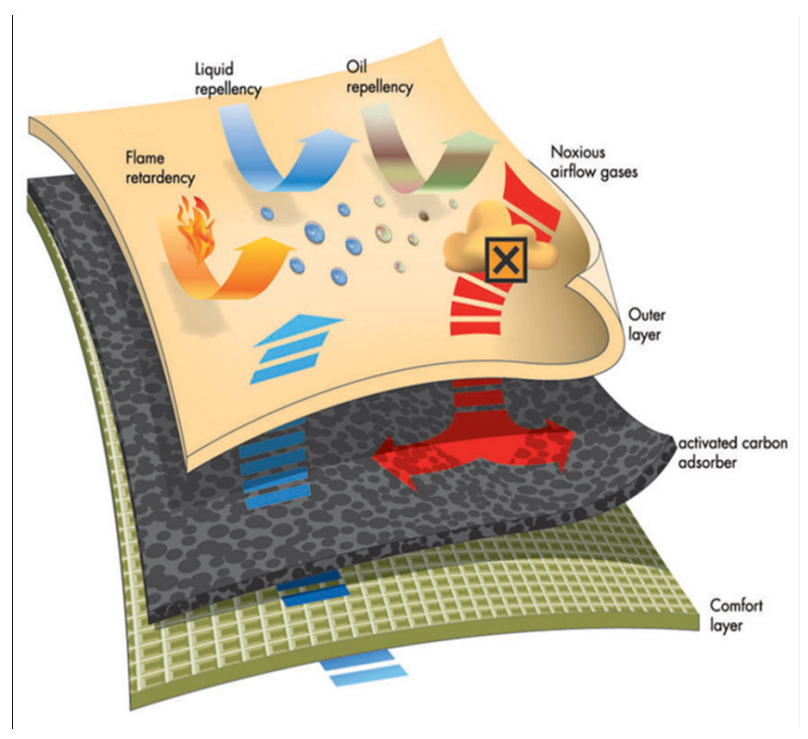

considered: the materials and the design.

These issues were analyzed and evaluated through projects carried out within the Scientific Research Center for CBRN Defense and Ecology (CCSACBRNE) in 2015.

The criteria that led to the choice of materials used for the manufacture of CBRN protective equipment (figure 1) were:

- the outer layer should be a coated fabric, to provide some degree of impermeability to water, oil and petroleum products. The material should also be fireproof and have good mechanical properties;

- the intermediate layer should be an adsorbent material, based on activated carbon, which provides, together with the outer layer, the protection against toxic compounds in the form of droplets, vapors or aerosols;

- the inner layer must be made of a material with 3D structure, having the function of providing thermal comfort.

From the thermal burden point of view, the assembly of materials (outer layer + the intermediate layer + inner layer) must provide a thermal resistance and air permeability as high as possible and resistance to water vapor as low as possible.

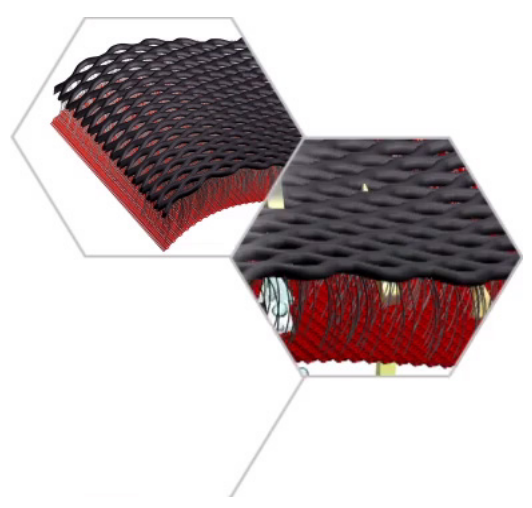

Figure 1. Structure of manufacturing materials of air permeable CB protective suits 


\section{Tools for thermal burden evaluation}

In order to measure the thermal burden of $\mathrm{CB}$ protective suits we can use the human volunteers. It is a valid but expensive and time-consuming approach, not to mention that the number of the tests should be high enough to eliminate any subjective evaluation. A more cost-effective and rapid approach is to be preferred.

By using standardized methods, in CCSACBRNE there have been evaluated mechanical, $\mathrm{CB}$ protection and thermal comfort parameters for various combinations of new generation materials used in manufacturing of air permeable protective suits.

Any new personal protective equipment (PPE) must therefore be evaluated to ensure that it poses lower thermal stress than the current standard for the same level of hazard protection. [1]

As outer layer have been used more than ten fabrics made from mixed fibers (cotton, modacryl, polyester, paraaramide or polyamide fibers), treated for water and oil repellency and flame retardancy.

As intermediate layer five types of fabrics based on activated carbon were used in the structures analyzed.

For the inner layer there were used several types of fabrics: net type materials, mixed fabrics of polyester and cotton "Coolmax" type, and fabrics with spatial structure.

Depending on the individual characteristics of each of these materials, there were analyzed and evaluated a large number of combinations of multilayer structures. 12 of these combinations were considered representative, being coded according to the manufacturer and type of material.

To establish the thermal comfort of the considered multilayer structures heat resistance and water vapor resistance of each structure were evaluated. A high level of thermal comfort means a higher thermal resistance and a lower resistance to water vapor.

In order to achieve an objective comparison of these parameters we used a quantifiable dimensionless index: permeability index, defined by the equation [4]:

$$
I=\frac{K \times R_{c t}}{R_{e t}}
$$

where

$-I: \quad$ permeability index;

- $R_{c t}$ : thermal resistance under steady-state conditions, $\mathrm{m}^{2} \mathrm{~K} / \mathrm{W}$;

- $R_{e t}$ : water-vapour resistance, under steady-state conditions, $\mathrm{m}^{2} \mathrm{~Pa} / \mathrm{W}$;

- $K$ - constant, $60 \mathrm{~Pa} / \mathrm{K}$.

The range of $\mathrm{I}$ is from 0 (impermeable) to 1 (ideal). [3]

The dry and wet (evaporative) heat transfer through multiple-layered textile materials were measured using Guarded Hot Plate (GHP) testing equipment.

A sample of material $(50 \times 50 \mathrm{~cm})$ is placed on a temperature-controlled flat plate in a controlled environmental chamber, with measurements made according to the ISO 11092. The procedure is designed to simulate the heat transfer that occurs in the microclimate created between the human skin surface and the textile material (i.e., personal protective equipment), and through the material itself to the surrounding ambient atmosphere. An advantage of GHP testing is that it can quickly evaluate and rank a large number of similar materials.

Following the determinations made there were obtained values for permeability index between 0.29 and 0.55 (figure 2).

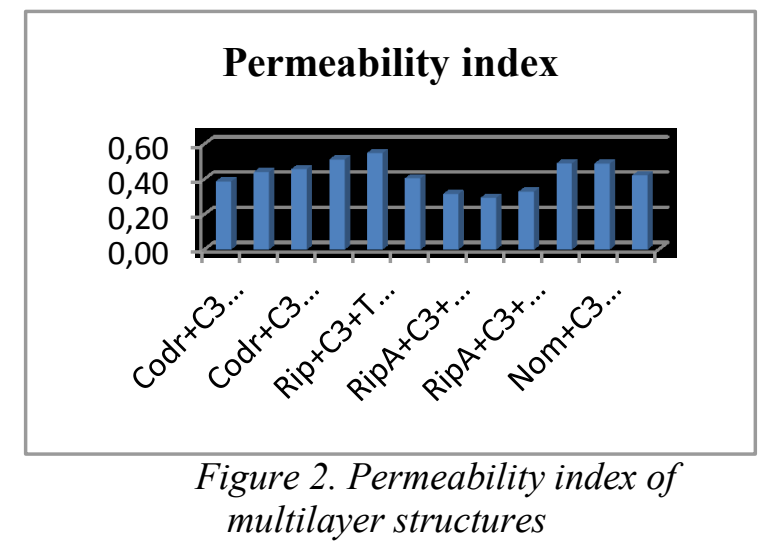




\section{Design of low thermal burden air permeable $\mathrm{CB}$ protective suits}

Based on the results obtained from experimental research on multilayer structures, a prototype batch of air permeable $\mathrm{CB}$ protective suits with lower thermal burden (figure 3) was produced.
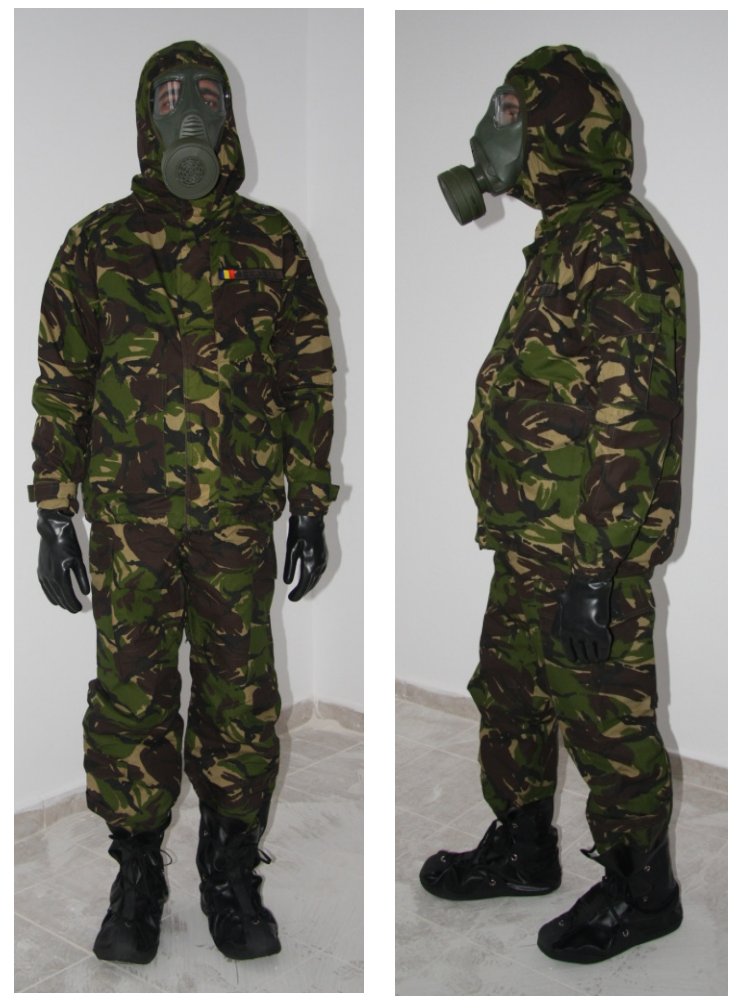

Figure 3. The air permeable $C B$ protective suit with lower thermal burden (ensemble)

The suit was made using the multilayer structure with the highest permeability index: a "Ripstop" fabric layer, flame retardant with hydrophobic and oleophobic treatment, that provides protection against droplets of warfare agents; as intermediate layer a fabric from activated carbon microfilaments, acting as a adsorbent of warfare agents in the form of vapors and aerosols; the inner layer, in contact with the wearer's skin, a 3D fabric, which works as a buffer layer between the body and the activated carbon material (figure 4).

The suit provides the same level of protection against chemical warfare agents as those existing in endowment.
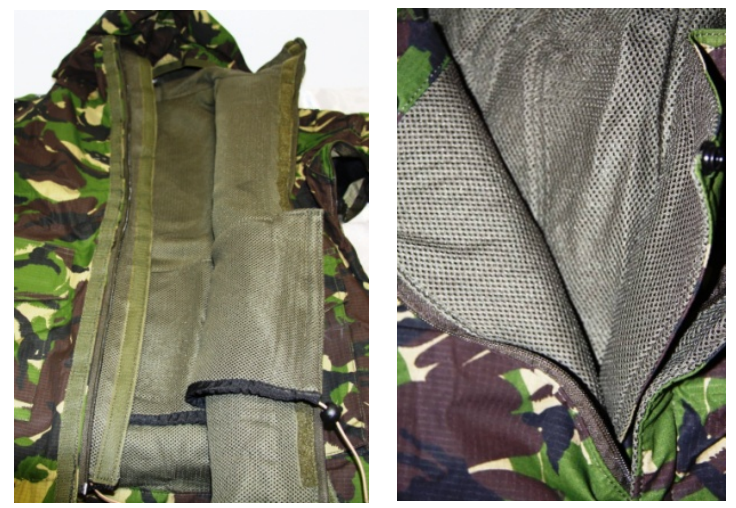

Figure 4. The inner layer based on a $3 D$ structure

In order to improve the thermal comfort, the suit was equipped with airtight zippers that can be opened during the use in "clean" environments, corresponding to MOPP 1-3 states. Zippers were placed in areas where accumulation of heat is likely to be met: armpits, back of knees, elbows (figure 5). The zipper role is to provide ventilation when opened (eliminating the accumulated heat), in closed condition unaffecting the role of the suit.
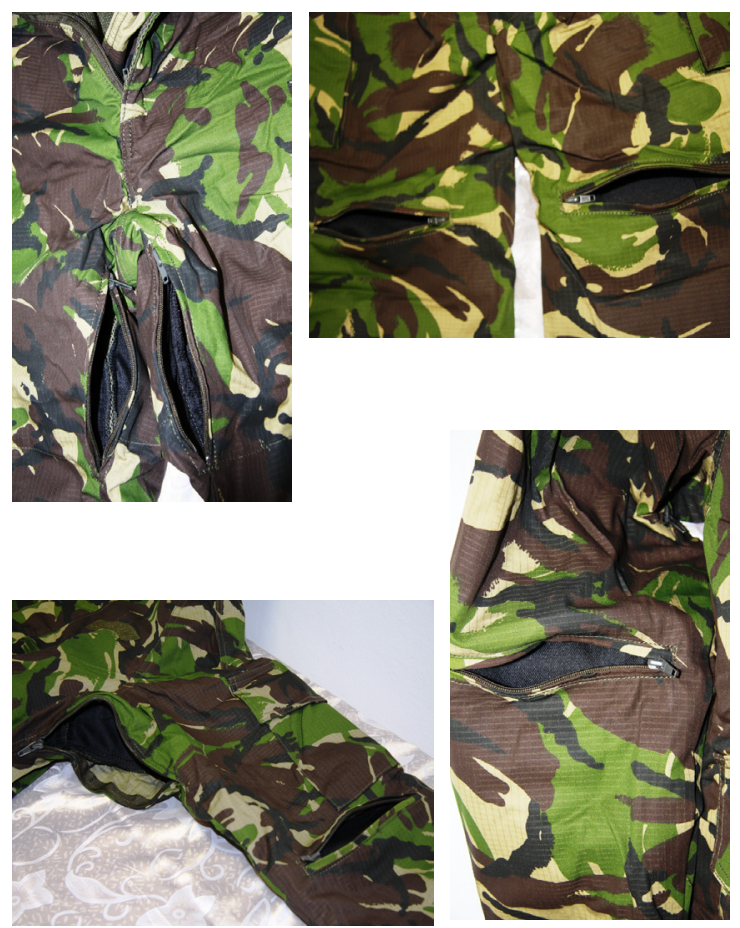

Figure 5. The airtight zippers on suits 
This kind of suit can be used by soldiers from all forces, protecting them from the effects of chemical, biological and radiological agents, in all climatic conditions, especially for warm and temperate climate areas, and also providing a higher thermal comfort than air permeable suits in use.

\section{Conclusion}

As a result of the conducted researches, Romania has an air permeable CB protective suit in accordance with the requirements of STANAG 4563, standard which were accepted and implemented from 2014.
Researching, developing and testing capabilities of CCSACBRNE have led to a new product that besides the new materials used in the manufacturing process also used new concepts of designing personal protective equipment.

The newly developed product provides the fighters that act in operational theaters with warm climates conditions an increased thermal comfort, with keeping the same protection capacity against chemical warfare agents.

\section{References}

[1] C. O'Brien, L.A. Blanchard, B.S. Cadarette, T.L. Endrusick, X. Xu, Larry G. Berglund, M.N. Sawka, R.W. Hoyt, Methods of Evaluating Protective Clothing Relative to Heat and cold Stress: Thermal Manikin, Biomedical Modeling, and Human Testing, Journal of Occupational and Environmental Hygiene, 8: 588-599, 2011.

[2] STANAG 4563, Tropical field clothing system (climatic zones B1, B2, B3)

[3] R.F. Goldman, B. Kampmann, Handbook on clothing. Biomedical Effects of Military Clothing and Equipment Systems, 2007.

[4] ISO 11092:2014, Textiles. Physiological effects. Measurement of thermal and watervapour resistance under steady-state conditions (sweating guarded-hotplate test). 